\title{
Stilwert und stilistische Funktionen von Vergleichskonstruktionen in Alma M. Karlins Werk ,Windlichter des Todes. Ein Roman aus Siam‘ (1933)
}

Inge POHL

\begin{abstract}
The stylistic value and functions of comparative structures in Alma M. Karlin's novel Windlichter des Todes. Ein Roman aus Siam (1933)

By analyzing specific comparative structures, the article demonstrates how Karlin uses this syntactic-structural model quantitatively and stylistically in the above-mentioned novel. Based on the results of this analysis and considering the potential recipients of Karlín's works, the paper characterizes the stylistic functions of these structures. It shows how specific stylistic features demonstrate Karlin's linguistic awareness and instincts.
\end{abstract}

Keywords: Alma M. Karlin, linguistic creativity, stylistic figure comparison, stylistic value, stylistic functions of comparison

DOI: $10.15452 /$ StudiaGermanistica.2021.29.0001

\section{Begriffliches, Methodisches und Ziel der Untersuchung}

Vergleiche stellen in belletristischen Werken Alma M. Karlins ein relevantes Stilelement dar, wie z. B. im Untersuchungsroman, dessen Handlung in Siam (heute Thailand) spielt: ${ }^{1}$

Wie Schneeflocken wirbelten weiße Falter über das satte Tropengrün. Auf und nieder, hin und her, wie Elfenfederbälle flogen sie im Lichtmeer des Tropentages. Weiß waren sie, wie Herbert Langes kummergebleichtes Gesicht, wie Primula Langfars Brautkleid, das unter liebenden Mutterfingern wurde, wie die Marmorstufen vor der Halle des Entzückens, in der Prinzessin Tup Tim sich in ihr Los zu fügen trachtete; weiß wie das zurückgeworfene Mückennetz, um Klementine Andersens Bett (WT 161).

Mit den zitierten Vergleichen beschreibt ein auktorialer Erzähler (nachfolgend aE) mittels verschiedenartiger Bildspender einen Ausschnitt aus der Natur Siams (weiße Falter). Sprachsensibel

Alle sprachlichen Romanbelege sind kursiv gesetzt. Der Bildempfänger ist in den Vergleichskonstruktionen unterstrichen, der Bildspender fett markiert. WT steht für den Titel des Untersuchungsromans mit nachfolgender Seite des Belegs. Damit es nicht zu Übercharakterisierungen kommt, bleiben syntaktisch beschriebene Elemente unmarkiert. 
nutzt die Schriftstellerin Karlin für die weiße Farbe der Falter vergleichende Bildspender aus dem Erfahrungsbereich europäischer Leser (wie Schneeflocken), vor allem jedoch komplex-anaphorisch emotional aufgeladene Bildspender aus dem literarischen Werk selbst. Lexeme, wie Elfen, Kummer, Brautkleid, liebend, Mutter, Entzücken, besitzen per se eine emotionale Aufladung, die sich innerhalb der Vergleiche textrelevant potenziert. Es geht im o. g. Textbeleg nicht um einen didaktisch orientierten Verstehensprozess, etwa einen Sachvergleich, sondern die differenzierten Formen und Funktionen des Stilelements Vergleich unterstreichen den literar-ästhetischen Anspruch des Werks von Alma M. Karlin. ${ }^{2}$

Der deutschsprachige Roman der slowenischen Schriftstellerin erschien 1933, in 1., 2. und 3. Auflage im gleichen Jahr. Mit dem Titellexem Roman hat Karlin eine explizite Gattungs-Kategorisierung vorgenommen, dadurch sind der Text und seine Funktion typisiert (vgl. Sandig 2006:318), eine fiktionale narrative Themenentfaltung mit einer charakteristischen Struktur wird erwartet. Inhaltlich (= Textbedeutung) geht es um (Liebes-)beziehungen zwischen Männern und Frauen im interkulturellen Bangkok, der Hauptstadt des Königreichs Siam. In der Textbotschaft orientiert Karlin auf die Thematik der „Rassenmischung“, ${ }^{3}$ indem exemplarisch vorgeführt wird, dass nur „reinrassige“ partnerschaftliche/eheliche Verbindungen glücklich werden können, wie die Liebesheirat der literarischen Figuren Primula Langfar und Dr. Norbert Nathfield. „Gemischtrassige“ Verbindungen im Roman sind zum Scheitern verurteilt und enden gar mit dem Tod eines Partners, wie der Halbsiamesin Klementine, die eine Liaison mit dem Deutschen Herbert Lange ersehnt, jedoch an ,gebrochenem Herzen“" und Cholera verstirbt (vgl. Tabelle in Pohl 2019a): ${ }^{4}$

\begin{tabular}{|l|l|}
\hline $\begin{array}{l}\text { Glücken } \\
\text { „reinrassiger“ Verbindungen }\end{array}$ & $\begin{array}{l}\text { Scheitern } \\
\text { „gemischtrassiger“ Verbindungen }\end{array}$ \\
\hline $\begin{array}{l}\text { Primula Langfar: Liebesheirat von Dr. Norbert } \\
\text { Nathfield }\end{array}$ & $\begin{array}{l}\text { Klementine Andersen: Ablehnung durch den Deutschen } \\
\text { Herbert Lange, Tod }\end{array}$ \\
\hline $\begin{array}{l}\text { Emma Friedländer: zeitlich begrenzte Geliebte } \\
\text { des Multimillionärs Moses Ehrental }\end{array}$ & $\begin{array}{l}\text { Anna Rüdiger: Heirat eines Malaien, Konvertierung zum } \\
\text { Islam, Absinken in der sozialen Stellung, wird Zweitfrau }\end{array}$ \\
\hline $\begin{array}{l}\text { Helene Freiwald: Entscheidung zur Heirat von } \\
\text { Hendrik van Haag }\end{array}$ & Fräulein d'Albuquerque: bleibt ehelos und kinderlos \\
\hline $\begin{array}{l}\text { siamesische Prinzessin Bhinarati: Traditions- } \\
\text { heirat eines siamesischen Prinzen }\end{array}$ & \\
\hline
\end{tabular}

Tab. 1: Partnerbindungen im Roman

Allgemein bekannte Komplikationen bei der Partnersuche - auf der Ebene der Textbedeutung kristallisieren sich im Roman deutlich heraus. Die Textbotschaft bezüglich „Rassenmischung“ vs. „Rassereinheit“ gibt sich nicht vordergründig zu erkennen, sie kann von Rezipienten nur anhand des Glückens oder Scheiterns der beschriebenen Paarbeziehungen inferiert werden. Ein individuelles Merkmal des Karlinschen Romans sehe ich darin, dass sich Vergleiche nur bezüglich der Textbedeutung, nicht aber bezüglich der Textbotschaft ausmachen lassen.

Dieser Aufsatz erweitert die Reihe von linguistischen Untersuchungen zum gleichen Werk der Autorin (vgl. Publikationen von Pohl im Literaturverzeichnis).

3 Vgl. die framesemantische Untersuchung zum Handlungsnomen „Rassenmischung“ im Untersuchungsroman in Pohl (2019a).

4 In der nachfolgenden Beschreibung sind diese literarischen Figuren benannt, darüber hinaus der in Siam lebende Deutsche Rüdiger und der auktoriale Erzähler $=\mathrm{aE}$. Aus sprachökonomischen Gründen benutze ich i. d. R. nur einen Namensteil der Figuren, Rüdiger und d'Albuquerque sind Familiennamen.

5 Das von Karlin durchgehend verwendete Fräulein bei d'Albuquerque in der Bedeutung ,kinderlose ledige Frau“ ist heute als veraltet zu lesen (vgl. DUW 2015:639). 
Vergleiche gehören unter stilistischem Aspekt zu den Tropen, sie bestehen aus drei Komponenten: Vergleichsobjekt - tertium comparationis - Vergleichsspender. Für die Begriffe „Vergleichsobjekt“ und „Vergleichsspender" finden sich in der Literatur verschiedene Äquivalente: Bild - Gegenbild, Bildempfänger - Bildspender, das Eigentliche - das Uneigentliche, Proprium - Improprium, hier verwende ich die geläufigen deutschen Bezeichnungen Bildempfänger und Bildspender. Die lateinischen Bezeichnungen gehen auf die antike Rhetorik zurück und gelten ursprünglich für die Komponenten der Metapher (vgl. Skirl/Schwarz-Friesel 2007:12). Formal und semantisch unterscheiden sich Metapher und Vergleich. Bei einer Vergleichskonstruktion bleiben im Unterschied zur Metapher die Benennungen für Bildempfänger und Bildspender nebeneinander bestehen: ein Gesicht wie ein Lederapfel zur Weihnachtszeit (WT 32). Die Vergleichskonstruktion ergibt keinen logischen Widerspruch, muss vom Rezipienten jedoch konzeptuell-semantisch gedeutet werden: das GESICHT IST WIE EIN LEDERAPFEL ZUR WEIHNACHTSZEIT BEZÜGLICH DER MERKMALE XYZ. Für ein erfüllendes Leseerlebnis sind Rezipienten gefordert, die Merkmale XYZ, worin Bildspender und Bildempfänger vergleichbar sind, zu inferieren. Die semantische Beziehung des Vergleichs wird im Gegensatz zur Metapher für den Rezipienten sichtbar an der sprachlichen Oberfläche ausgeführt, in der Regel mit den beiden Komponenten Bildempfänger und Bildspender sowie einem Vergleichssignal. Bildempfänger und Bildspender gemeinsam stellen das semantische Konstrukt der Vergleichskonstruktion dar, das insbesondere bei der Beschreibung stilistischer Funktionen zu berücksichtigen ist (vgl. Punkt 4.).

In der Analyse ist zwischen der syntaktischen Ebene der Satzglieder und der semantisch-stilistischen Ebene von Bildspender und Bildempfänger zu unterscheiden. Semantisch erstreckt sich der mit wie eingeleitete Bildspender auf denjenigen Bereich in der Basis, der ,eine zum VergleichsAdjunkt passende Bedeutung“" aufweist (Weinrich 1993:787). Insofern kann sich ein Bildspender, der syntaktisch als Komparatives Adverbiale dem Prädikat untergeordnet ist, semantisch auf ein anderes Satzglied beziehen, vgl. die Brüste der Eurasierin wuchsen wie Wellen bei losbrechendem Sturm (WT 24) - der Bildspender (Wellen bei losbrechendem Sturm) erstreckt sich semantisch auf das Satzsubjekt als Bildempfänger (die Brüste der Eurasierin).

Ein tertium comparationis (t. c.) erfasst das/die (angenommene/n) gemeinsame/n, vergleichba$\mathrm{re} / \mathrm{n}$ Merkmal/e XYZ von Bildempfänger und Bildspender. Es bietet sich nicht immer verbalisiert an, sondern muss über die kognitiv-semantische Analyse von Bildempfänger und Bildspender inferiert werden (Burger, 2007:48, gibt ein fehlendes t. c. als typisches Merkmal okkasioneller Vergleiche an). Im Beispiel ein Gesicht wie ein Lederapfel zur Weihnachtszeit (WT 32; aE über Klementines Großmutter) ist das t. c. mit den Semen ,faltenreich', ,mit Einkerbungen und Flecken', ,bräunliche Farbe', ,ledrige Konstitution' beschreibbar. Da ich in meiner Untersuchung die denotativ-semantische Herkunft von Bildspender und Bildempfänger sowie den ,semantischen Sprung“ zwischen beiden fokussiere, bleibt das t. c. weitestgehend unberücksichtigt.

Theoretisch ist der syntaktische Status von Wortgefügen mit wie problematisch. Zwischen den Elementen der Vergleichskonstruktion wie + Bildspender bestehen nicht die für eine Wortgruppe typischen Merkmale der Subordination. Andererseits sind die Vergleichssignale (wie, so ... wie) nicht nur grammatische Hilfswörter, ${ }^{6}$ sie indizieren die semantische Beziehung des Vergleichens zwischen sprachlichen Einheiten. ${ }^{7}$ Der geläufige Begriff „Vergleichspartikel“ ist ebenfalls zu hinterfragen, denn die Weglassbarkeit von Partikeln, ohne dass die Sachverhaltsbeschreibung gestört wird, ist nicht gegeben. Trotz der bestehenden terminologischen Unklarheiten verwende ich die Begriffe „Wortgruppe“ und „Vergleichspartikel“.

\footnotetext{
Üblicherweise werden grammatische Hilfswörter nicht als Lexeme betrachtet, so dass die Verbindungen von grammatischen Hilfswörtern mit Autosemantika als ein Wort aufgefasst werden, wie Artikel + Nomen, temporales Hilfsverb + Vollverb bei der Konjugation, Adjektiv + Hilfswort bei der Steigerung.

7 Karlin verwendet nur einmal eine schriftsprachlich nicht korrekte Vergleichspartikel: Bah! Affen hatten bessere Hände wie das alberne Fürstenkind (WT 155; Zweitfrau des siamesischen Fürsten über Bhinarati) beim Attribut zum Akkusativobjekt (haben ,besitzen'). Gegenwartssprachlich korrekt wäre: bessere Hände als das ... Möglicherweise charakterisiert Karlin damit das Sprachporträt der siamesischen Zweitfrau.
} 
Bildspender nutzt Karlin in einfachen, vor allem jedoch in erweiterten Strukturen. Beim einfachen Vergleich besteht die bildspendende Wortgruppe lediglich aus wie + Einzelwort: Wie Schneeflocken wirbelten weiße Falter (WT 161; aE über die Erzählsituation). Beim erweiterten Vergleich wird der Bildspender formal angereichert, z. B. wie + nominale Wortgruppe: eine Pause, in der sie feindlich abwehrende Gedanken wie gezückte Dolche fühlte (WT 9; aE über d'Albuquerque) oder wie + präpositionale Wortgruppe: ,, Me ... Me ... “ wiederholte sie wie zu einem Kinde (WT 36; aE über Klementine). Schließen sich an den Bildspender Nebensätze an, liegen ebenfalls erweiterte Vergleiche vor, z. B. wie + Pronomen + Teilsatz: Klementine Andersen ging die Suriwongsestraße entlang wie jemand, der die Welt an der Leine hat (WT 78; aE über Klementine) oder wie + Nomen + Teilsatz: Wie Nebel, der nur noch die fernsten Berge verhüllt und mehr und mehr Bäume des Flachlands freigibt, zeigten sich ihr, obschon vom Augenblicksrausch getrübt, die Bilder der Vergangenheit (WT 103; aE über die Gefühlslage Emmas nach der Werbung durch Herbert Lange). Karlin schöpft aus einem reich vorhandenen sprachlichen Reservoir an (Bildspender-)Formen und setzt dieses variabel bei betonter Bevorzugung erweiterter Vergleiche ein.

Auf die stilistische Relevanz der Karlinschen Vergleiche verweisen neben der Verwendungsquantität die unterschiedlichen sprachlichen Formen zum Ausdruck eines Vergleichs sowie die Kreation überwiegend okkasioneller Vergleiche. Auf den Romanumfang von 177 Seiten verteilen sich die ermittelten 141 Vergleichskonstruktionen im obigen Verständnis mit beinahe je einem Beleg pro Seite. Über Vergleichskonstruktionen hinaus dienen Karlin verschiedene Wortarten mit dem Sem ,ähnlich sein` oder ,prüfend gegeneinander abwägen` zum Ausdruck des Veranschaulichens:

- das Verb gleichen: „Manche Leute bleiben im Schoß der Familie; sie gleichen Schiffen, die am Hafendamm liegen "(WT 124; Dr. Nathfield zu Primula);

- das Präfixverb vergleichen: Die Letzte der d'Albuquerque [...] verglich sich innerlich mit einem Schwamm und ihre Mitmenschen mit Steinen (WT 83; d'Albuquerque über sich selbst);

- die gehobensprachliche Präposition gleich (+ Dativ): und jene Handlungen unzweifelhaft die besten waren, die sich gleich Pflastersteinen in das Gefüge ringsumher einschoben (WT 145; Emma über das menschliche Leben und die Menschen);

- das Adverb gleichsam: Tup Tim erhob sich, gleichsam gealtert, ernst, würdevoll (WT 98; aE über die siamesische Prinzessin Tup Tim, nachdem der Vater sie zu einer Traditionsheirat zwingt);

- das emotional verstärkende Adverb sondergleichen: Der Weg zur Brücke, ein Gerümpel sondergleichen, über das man seiltänzerartig hinwegklettern mußte, war schlüpfrig, unsicher (WT 149; aE über ein Wohngebiet in Bangkok, in welchem Anna wohnt).

Karlin nutzt zudem Möglichkeiten der Wortbildung, wie das Wortbildungssuffix -haft mit einer Tierbezeichnung als Basis: katzenhaft weich tastete die braune Hand (WT 5; aE über Fräulein d'Albuquerque) oder die Komposition (Nomen + Präposition), wie bei (Emma) und ihre untersuchungsrichtergleichen Fragen (WT 75; aE über Emma).

In der vorliegenden Untersuchung wird nach der literar-ästhetischen Rolle lediglich der Vergleichskonstruktionen gefragt. Es werden deren Stilwerte herausgearbeitet, stilistische Informationen, die ,nicht mit den sprachlichen Mitteln an sich gegeben sind, sondern relational bedingt sind“ (Fleischer/Michel/Starke 1993:50; vgl. Sandig 2006). Hier betrachte ich die dominierenden stilistischen Funktionen von Vergleichskonstruktionen im Relationsgefüge von Autorin, Werk und potenziellen Rezipienten. Stilistische Funktionen, wie Selbstdarstellung, Wertung, Textauflockerung usw. basieren auf dem Stilwert von Stilelementen, der variabel sein kann. Davon auszugehen ist, dass das polyfunktionale Stilelement des Vergleichs verschiedene Stilwerte einbringt, die insbesondere durch das semantische Zusammenspiel von Bildempfänger und Bildspender konstituiert werden. In der Synthese mit weiteren Stilelementen tragen Vergleichskonstruktionen zur Stilgestalt dieses Romans bei (vgl. Pohl 2019a, b, c; 2020). Im Zentrum meiner Untersuchung stehen folgende Ziele: Aufzudecken sind die strukturell-semantischen Besonderheiten der Karlinschen Vergleichs- 
konstruktionen nach Quantität und Qualität. Es ist herauszufinden, welche Denotatsbereiche Karlin sowohl für Bildspender als auch für Bildempfänger wählt. Aus den Analyseergebnissen sind die stilistischen Funktionen der Vergleichskonstruktionen abzuleiten. Schließlich sind die Eigenheiten Karlins bezüglich der verwendeten Vergleichskonstruktionen zu erhellen sowie erkennbare individualstilistische Besonderheiten vorsichtig zu formulieren.

\section{Analyse der syntaktischen Strukturen der Vergleichskonstruktionen}

Die Fachliteratur bietet zwar verschiedene Klassifizierungsmöglichkeiten für Vergleichskonstruktionen an, ein hilfreiches Analyseraster liegt nicht vor. So spricht Weinrich von der „Offenheit des Vergleichs", weil man inhaltlich Beliebiges miteinander vergleichen kann, weil alle Arten sprachlicher Zeichen die Vergleichskomponenten besetzen und unterschiedliche Satzglieder im Skopus (Erstreckungsbereich) des Vergleichs stehen können (vgl. 1993:785ff.). Bei meiner syntaktischen Analyse wähle ich den Bildspender als Kategorisierungs-Kriterium. Ermittelt wird, welche verschiedenartigen syntaktischen Strukturen Karlin nutzt und in welcher Frequenz diese gebraucht sind. Aufgrund dessen, dass Karlin den Bildspender entweder in einem Teilsatz oder in einem Satzglied/ Satzgliedteil verbalisiert, ergibt sich eine quantitative Rangreihe für die ermittelten 141 Belege: Modaladverbiale: 94, Attribute: 34, Prädikative: 9, komparative Nebensätze: 4 Belege.

\subsection{Analyse der syntaktischen Strukturen der Bildspender in Form von Satzgliedern und Satzgliedteilen}

\subsubsection{Der Bildspender in der syntaktischen Form des Modaladverbiale}

Bekanntlich ,veranschaulichen“ Modaladverbiale, inkl. das Komparative Adverbiale, das im Prädikat ausgedrückte Geschehen, indem eine bestimmte Eigenschaft des Geschehens fokussiert wird. $\mathrm{Zu}$ erfragen ist aus stilistischer Sicht die Begrifflichkeit von „Veranschaulichen“, ist ein „Bild zum Anschauen“"gemeint? Definitiv kommen beim Veranschaulichen auch andere Sinne als der optische Sinn zum Zug. Unter ,Veranschaulichen“ verstehe ich deshalb, dass ein Geschehen den menschlichen Sinnen zugänglich gemacht wird, dass Rezipienten sinnfällig einbezogen werden. Üblicherweise werden fünf Sinne des Menschen unterschieden, mit denen er die Umwelt physiologisch wahrnehmen kann. Hier unterscheide ich die Sinne des Sehens, Hörens, Riechens, Schmeckens, Tastens und aufgrund des Materials zusätzlich die Empfindung (Begrifflichkeiten und Beispiele vgl. unten). Bei den Karlinschen Komparativen Adverbialen spielen die Sinne des Riechens und Schmeckens keine Rolle, die Autorin spricht in absteigender Rangreihe die Sinne des Sehens, der Empfindung, des Hörens und Fühlens an.

Der visuellen Wahrnehmung wird im Roman größte Bedeutung beigemessen, indem Vergleiche das im Prädikat ausgedrückte Geschehen ,vor dem inneren Auge“ des Rezipienten sichtbar werden lassen. Sehen gilt bekanntermaßen als wichtigste Sinneswahrnehmung des Menschen. So werden „unsichtbare“ Gefühle einer literarischen Figur über den Vergleich mit Konkretem „sehbar": Helene fühlte sich wie ein Bambus im Sturm (WT 57; aE über Helene, als sie von den Heiratsabsichten des deutschen Witwers Sturpopp erfährt). Mehrheitlich jedoch will Karlin die exotische Umgebung in Siam für den europäischen Leser optisch vorstellbar werden lassen. So wird „Fremdes“ mit bekanntem Sichtbaren verglichen: (Herbert Langes Blick) huschte hierauf wieder uferwärts zu den komischen Wasserhäusern, die offen waren wie aufgeklappte Holzschachteln (WT 18; aE beschreibt Bangkok). Weniger rezipientenfreundlich wirken allerdings diejenigen Vergleiche, in denen als Bildspender die den europäischen Lesern unbekannte Natur Siams gewählt wird: Fräulein d'Albuquerque klebte förmlich auf dem Stuhl wie eine vom Tropenschauer breitgeschlagene, braungelbe Alimanderblüte (WT 179; Frau Langfar über d'Albuquerque).

Empfindung spielt im Untersuchungsroman die zweitgrößte Rolle. Darunter verstehe ich ,innere" Wahrnehmungen, abstrakte Sinneseindrücke, die im seelisch-geistigen Bereich anzusiedeln 
und mit einem (Kontext-)Sem ,wertend' gekoppelt sind. Die Grenze zu Emotionen ist unscharf: Klementine folgte wie im Traum, stolperte dahin, ohne Hindernisse zu beachten (WT 148; aE über Klementine, nachdem Herbert Lange sie abgelehnt hat).

An dritter Stelle rangieren im Roman Vergleiche, die das prädikative Geschehen für einen Rezipienten auditiv wahrnehmbar umsetzen. Oft handelt es sich um die akzentuierte Beschreibung von Hörbarem, welches durch Vergleich mit Bekanntem semantisch spezifiziert wird, z. B.: Wie ein Jubelruf brach die Antwort von ihren Lippen (WT 123; aE über Primulas Antwort zum Heiratsantrag von Dr. Nathfield). Wiederholt geht es Karlin jedoch darum, die siamesische Umgebungssituation hörbar zu veranschaulichen: des allgemeinen Lärms des Ostens, der wie der Wellenschlag an rauher Küste kein Abnehmen kennt (WT 49; aE über Siam).

Eine taktile Wahrnehmung des Geschehens vermitteln nur wenige Vergleiche, z. B. weil sie (Primula - I.P.) die Augen des zweiten, ihr unbekannten Weibes wie Nadelstiche auf dem Gesicht fühlte, beschlich sie ein wachsendes Unbehagen, eine Vorahnung von etwas ganz Bösem (WT 151; aE über Primula und die Erstfrau Ibrahims).

Die stilistische Funktion des Veranschaulichens, die u. a. auf den Stilwerten der Modaladverbiale beruht, durchzieht den gesamten Roman - Karlin spricht verschiedenste Rezipienten-Sinne an, wobei der optische Sinn dominiert.

\subsubsection{Der Bildspender in der syntaktischen Form des Attributs}

Die Bildspender-Attribute bezieht Karlin morphologisch-syntaktisch vielgestaltig auf Nomen, Pronomen oder Adjektive bzw. adjektivisch gebrauchte Partizipien, die jedoch nur beim Bezugswort in der Form des Nomens den Bildempfänger benennen, wie beim Beispiel: ein Kleid von diesem warmen Gelbrot wie ein Sonnenuntergang zur Trockenzeit (WT 86; Fräulein d'Albuquerques Kleiderwunsch). Mit Vorliebe kombiniert die Autorin das Stilelement des Vergleichs mit anderen Stilelementen. So sind beispielsweise Eindrücke verschiedener Sinne synästhetisch verschmolzen, hier der Empfindung (Gefühl) und des Sehens (Gedankenstrich): in abgebrochenen, zittrigen Sätzen, wie Gefühlsgedankenstriche, kamen die Einzelheiten (WT 41; aE über Helene, die einen Heiratsantrag erhält).

Bei Bildspendern, die als Attribute zu adjektivischen oder pronominalen Prädikativen sowie adjektivischen Adverbiale auftreten, steckt das t. c. regelgemäß, folglich für Rezipienten leicht erschließbar, im syntaktischen Bezugswort des Attributs, z. B. beim adjektivischen Prädikativ grau: Grau, wie die Hufeisengräber des angrenzenden Chinesenfriedhofes waren auch hier die Steine (WT 177; aE über den Friedhof, auf dem Klementine liegt). Ebenso ist im partizipial-adjektivischen Adverbiale (rotglänzend) als Bezugswort des Attributs das t. c. verbalisiert: Tränen, Schweiß, getauschte Küsse machten Helene Hauswalds Gesicht rotglänzend wie eine Lackschale (WT 171; aE über Helene bei der Abfahrt zu ihrem Geliebten).

Rezipienten werden kognitiv stärker gefordert, wenn das t. c. selbst nicht nur unidirektional erschlossen werden muss: $i$ ch bin blank wie ein Soldatenknopf (WT 9; Helene über ihre Finanzen). Karlins Formulierung ist insofern bemerkenswert, als die phraseologische Bedeutung von blank sein: ugs. ,kein Geld mehr haben“ (DuR 2008:124) und die wörtliche Bedeutung von blank sein, ein Gegenstand ,glänzt', kontaminiert sind. Ein Rezipient muss zwischen semantischen Ebenen switchen können, um den Vergleich insgesamt zu verstehen: die hyperbolisch-expressive Verstärkung der Semantik des ,Kein-Geld-mehr-Habens'.

Karlin nutzt wiederholt das pronominale Prädikativ, um Bildspender-Attribute anzuschließen, z. B. zum Indefinitpronomen etwas: Liebe ist etwas wie der Abendstern, der nie untergeht (WT 64; Moses über die Liebe) - die Emotion Liebe wird mittels eines Vergleichs dem optischen Sinn von Rezipienten zugänglich gemacht. Der Vergleich selbst könnte ohne das Indefinitpronomen angeschlossen werden (z. B. Liebe ist wie der Abendstern). Durch das Vorschalten des Pronomens erhält die Vergleichskonstruktion formales und semantisches Gewicht: Die literarische Figur Moses Ehrental kann der Aussage das Sem der,Vagheit“/`Offenheit‘/'Unbestimmtheit' hinzufügen. 
Eine stilistisch individuelle Verwendung des Bildspender-Attributs findet sich beim prädikativen Attribut braun: Ehe Primula zuzugreifen vermochte, war die Gefährtin unten im Klong, tauchte braun wie eine Schlammnixe wieder auf; tropfte grünschleimig ans Ufer (WT 149; aE über den Sturz von Klementine in einen Klong). braun ist hier das Merkmal eines Lebewesens (Subjekt des Satzes die Gefährtin), das nur während des vom Verb ausgesagten Geschehens tauchte auf gilt - das Bildspender-Attribut (wie eine Schlammnixe) visualisiert das Geschehen und spezifiziert die Farbe braun semantisch.

Bei attributiven Vergleichs-Anschlüssen liegt möglicherweise Karlins Intention zugrunde, das t. c. rezipientenzugewandt zu verbalisieren bzw. das t. c. unterhaltungsintendiert-sprachspielerisch einzusetzen.

\subsubsection{Der Bildspender in der syntaktischen Form des Prädikativs}

Das mit wie verbundene Prädikativ bildet in den Belegen zusammen mit einem Kopulaverb (hier sein, werden, dünken $)^{8}$ das Prädikat eines Satzes. Alle von Karlin eingesetzten Prädikative referieren semantisch auf ein Merkmal des im gleichen Satz benannten Subjekts, das den Sinnen zugänglich gemacht wird und im Vergleich implizit ein Urteil/eine Einordnung präsupponiert. In der Fachliteratur sind Vergleichskonstruktionen in der Form von Prädikativen seltener genannt - bei Karlin scheint es (in verschiedenen Wortarten verbalisiert) ein individualstilistisches Merkmal zu sein.

Verwendet Karlin für das Prädikativ ein Nomen (mit Attributen): ,Oh, ich bin wie das Kamel in der Wüste, das beim letzten Stroh zusammenbricht ..." (WT 85; d'Albuquerque über sich selbst), so wird ein (pejoratives) Werturteil über die hier im Subjekt genannte literarische Figur gefällt. Karlin nutzt ebenso die Möglichkeit, die im Subjekt genannte literarische Figur in eine (pejorativ bewertete) Begriffsklasse/Gattung einzuordnen: ,, Sie sind wie alle Frauen, Emmachen; Wahrheit ist Ihnen lästig“" (WT 132; Moses Ehrental macht Emma den Vorschlag, seine Geliebte zu werden).

Mit prädikativen Adjektiven (mit Attributen) hebt Karlin ein Merkmal/eine Eigenschaft der im Subjekt genannten Figur hervor, z. B.: ,Entschuldigen Sie, Fräulein Andersen, ich wußte nicht, was ich tat! Der Schmerz, ich ... ich ... war wie wahnsinnig ... “ (WT 142; Herbert Lange, nachdem er Klementine geküsst hat).; wahnsinnig sein bedeutet ugs ,nicht recht bei Verstand sein'.

Individualstilistisch fällt auf, dass Karlin das Prädikativ häufig mit Attributen detailreich ausbaut. Diese erweiterten Vergleiche vermitteln dem Rezipienten über den im Subjekt verbalisierten Sachverhalt mehr Informationen als einfache Vergleiche, vgl. Äußerlich war er wie ein Berg (konstruiertes Beispiel) vs. Äußerlich war er wie ein Berg, den Regengüsse abgeschürft haben, bei dem man jedoch im schroffen Gestein oft unerwartet auf reiche Edelerzadern stößt (WT 47; aE über Rüdiger). ${ }^{9}$

\subsection{Analyse der syntaktischen Strukturen der Bildspender in Form von komparativen Ne- bensätzen}

Karlin verwendet vergleichsweise wenige komparative Nebensätze (nachfolgend NS), diese werden mit wie / als / als ob eingeleitet, ihre stilistische Grundfunktion besteht im Veranschaulichen im o. g. Sinn. So visualisiert ein realer Vergleich (mit Indikativ) den im Hauptsatz genannten Vorgang: ,, Wir sind zu Leid geboren, genau wie der Funke aufwärtsfliegt ... " (WT 179; d'Albuquerque über das menschliche Leben). Durch die Erweiterung der Vergleichspartikel wie mit der Partikel genau wird „eine ganze Vergleichsskala von der völligen Gleichheit bis zur völligen Ungleichheit eröffnet“ (Weinrich 1993:789; vgl. dort die Skala in Verbindung mit so: ganz genau so, genau so, ebenso, so, fast so, kaum so, längst nicht so + wie), genau gibt im Beispiel demnach nur eine relative Gleichheit

Karlin nutzt ebenfalls scheinen als Kopulaverb, nicht im Untersuchungsroman finden sich heißen und bleiben.

Stilistisch trifft auf die für Karlin typischen detailreichen Vergleiche der „Aspekt der Natürlichkeit“ zu: Je mehr Sprachmaterial verwendet wird, desto gewichtiger wird das Beschriebene (vgl. Sandig 2006:187). 
an. Auch beim nachfolgenden Prädikativsatz setzt Karlin einen realen Vergleich (mit Indikativ) zur Zustandsbezeichnung im Hauptsatz ein: Klementine war es, wie es einem manchmal ist, wenn man an einem kalten Wintertag plötzlich die windstille Sonnenfront eines Hauses gefunden ... (WT 36; aE über Klementine nach dem freundlichen Gruß der Großmutter). sein im Hauptsatz bedeutet ,sich in einer bestimmten Lage befinden; sich bestimmten Umständen ausgesetzt sehen ' (DUW 2015:1596). Die dem Rezipienten zunächst unbekannte Empfindung Klementines wird im Komparativsatz mit einer allgemein bekannten Empfindung veranschaulicht. Bildspendend sind ebenfalls komparative Attributsätze (Satzgliedteile) zu einem vorhergehenden Nomen (mit Attributen), die einen irrealen Vergleich (mit Konj. II) ausdrücken, z. B.: eine typische Eurasierin mit breiten, seltsam farblosen Lippen und unverkennbarem Schwingen der einzelnen Körperteile, als ob innere Gummibänder nachgelassen hätten (WT 71; Herbert Lange über „Mischlingsfrauen“).

Mit dem Einsatz von Komparativsätzen nutzt Karlin die stilistische Möglichkeit, Romanaussagen durch vergleichbare reale bzw. irreale, jedoch aus dem Alltag der Rezipienten bekannte Geschehenseinheiten semantisch zu veranschaulichen.

\section{Repräsentierte Denotatsbereiche bei Bildempfänger und Bildspender}

Für die nachfolgende Analyse wähle ich die bei Karlin dominierenden nominalen Vergleiche aus (133 von 141 Belegen), bei denen Bildempfänger und Bildspender durch Nomina gebildet sind. Folgende Fragen sind zielführend:

Aus welchen Denotatsbereichen stammen die Bildempfänger? Die Analyse zeigt, welche lexikalisch-semantischen Elemente aus welchen Denotatsbereichen Karlin als wert erachtet, sie mit einem Vergleich auszuschmücken, anschaulicher, den Sinnen zugänglicher zu machen u. Ä. Wie ist das quantitative Verhältnis der Denotatsbereiche zueinander?

Aus welchen Denotatsbereichen stammen die Bildspender? Wie ist das quantitative Verhältnis der Denotatsbereiche zueinander? Die Analyse erhellt, welchen Reichtum an lexikalisch-semantischen Elementen aus welchen Denotatsbereichen Karlin heranzieht, um den Bildempfänger stilistisch zu fokussieren. Bekanntlich hat eine Autorin/ein Autor vielfältigste Optionen, einen Bildspender zu wählen. Wählt Karlin tradierte Bildspender aus oder geht sie unkonventionell vor?

Bleibt Karlin mit Bildempfänger und -spender im gleichen Denotatsbereich bzw. dessen Subgruppen (homogener Vergleich) oder nutzt sie semantische Sprünge zwischen verschiedenen Denotatsbereichen und deren Subgruppen (heterogener Vergleich)? Letztlich wird sichtbar, welcher Art die Relationen zwischen Bildempfänger und Bildspender sind und ob sich bestimmte Muster feststellen lassen. Welchen Zusammenhang gibt es zwischen den von Karlin ausgewählten Denotatsbereichen von Bildempfänger und Bildspender und der Autorin-Intention?

Bei der Suche nach Denotatsbereichen, welche von den Bildempfängern repräsentiert werden, lasse ich mich von den vorgefundenen Belegen leiten. Es stellt sich erwartungsgemäß heraus, dass die Denotatsbereiche der Bildempfänger mit der Textbedeutung korrelieren: Der Roman handelt von Beziehungen zwischen Menschen in einer interkulturellen Situation, so dass sich die Bildempfänger in den Denotatsbereichen MENSCH, KULTUR, NATUR und deren Subgruppen wiederfinden.

Der Denotatsbereich MENSCH umfasst Bezeichnungen für die „,mit der Fähigkeit zu logischem Denken u. zur Sprache, zur sittlichen Entscheidung u. Erkenntnis von Gut und Böse ausgestatteten höchstentwickelten Lebewesen“ (DUW 2015:1188). Denotative Subgruppen ergeben sich aus dem Untersuchungsmaterial:

(1) Mensch/Eigenname/Pronomen: Die Bezeichnungen dieser Gruppe benennen literarische Figuren mit ihren Eigennamen (z. B. Klementine, Lange, Primula). Insofern Pronomen auf die genannten Figuren referieren, sind sie den entsprechenden literarischen Figuren zugeordnet.

(2) Mensch/Menschlicher Körper: Der Körper (inklusive seiner Teile) von literarischen Figuren initiiert die Vergleiche (z. B. Gesicht, Nase, Nabelschnur). 
(3) Mensch/Eigenschaft/Situation/kulturelle Herkunft: ${ }^{10}$ Die Bezeichnungen von literarischen Figuren sind durch charakterliche Eigenschaften, sich aus einer Situation ergebende Eigenschaften oder die kulturelle Herkunft motiviert (z. B. siamesische Nachbarin, die alte Siamesin, eine glückliche Braut).

(4) Menschliche Emotion: Die Lexeme orientieren auf die psychisch-physische Bewegtheit der literarischen Figuren, die sich aus komplexen Bewertungen des eigenen Körpers, der seelischen Befindlichkeit, seiner kognitiven Denkinhalte, der allgemeinen Umweltsituationen usw. ergeben (vgl. ausführlich Schwarz-Friesel 2007:49ff.) ${ }^{11}$ (z. B. Stolz und Bedauern, Liebe, glühender Kummer).

(5) Mensch/Gattung: In dieser Gruppe erscheinen Bezeichnungen für den Menschen als Gattungswesen (z. B. Menschen - im Pl.).

(6) Mensch/Beruf/Stand: Die Lexeme dieser Gruppe sind durch einen Beruf oder einen gesellschaftlichen Stand motiviert (z. B. Dienerinnen, Sultan, Koch).

(7) Mensch/Geschlecht: Die Geschlechtszugehörigkeit motiviert die Lexeme, d. h., Karlin nutzt geschlechtsspezifische sowie geschlechtsneutrale Lexeme (z. B. zwei Mädchen, die weiblichen Mitglieder des Hauses).

(8) Menschliche Empfindung: Die Lexeme benennen primäre Erfahrungs- bzw. Erlebenszustände, die sich kategorial von Emotionen unterscheiden (vgl. Schwarz-Friesel 2007:50). Es handelt sich um figurenbezogene Empfindungen (z. B. Eindrücke in: Wie Keulenschläge waren die neuen Eindrücke auf sie gefallen und hatten sie betäubt (WT 32; aE über Klementine; des Weiteren: das Ungewohnte - wie eine Springflut; etwas Düsteres - wie Wolken hinter Bergen).

Für den Denotatsbereich KULTUR lege ich folgende definitorische Bestimmung zugrunde: „Gesamtheit der geistigen, künstlerischen, gestaltenden Leistungen einer Gemeinschaft als Ausdruck menschlicher Höherentwicklung“" (DUW 2015:1077). Die Subgruppen ergeben sich aus dem Beispielmaterial:

(1) Geistige Kultur: Hierunter fasse ich alle Bezeichnungen, die sich auf „das Denkvermögen des Menschen, seine Verstandeskräfte, seine Fähigkeit, Dinge zu durchdenken u. zu beurteilen" (DUW 2015:694) beziehen (z. B. feindlich abwehrende Gedanken, die Zweifel). Denkbar wäre hier eine Zuordnung von Bezeichnungen für sprachliche Äußerungen, da Sprache und Kognition eine Einheit bilden. Da die konkreten Sprachäußerungen im Roman immer auch soziale Beziehungen zwischen den Figuren zum Ausdruck bringen, habe ich mich für deren Zuordnung zu Sozialer Kultur entschieden.

(2) Soziale Kultur: Unter Sozialer Kultur will ich „das (geregelte) Zusammenleben der Menschen in Staat u. Gesellschaft" (DUW 2015:1641) verstehen. In diese Gruppe nehme ich alle Bezeichnungen auf, die auf zwischenmenschliche Beziehungen referieren, z. B. alle Formen der menschlichen Kommunikation mit Sprache, Blicken usw. (z. B. die erregten Worte, hochtönendes, ununterbrochenes Geschwätz), auch sozial-kulturelle Gliederungen des Lebens (z. B. Jahre). Im Einzelfall ist die Grenze zur Geistigen Kultur unscharf.

(3) Materielle Kultur: Hier erfasse ich alle Bezeichnungen, die sich auf Materie beziehen, die „stofflich, dinglich, gegenständlich, körperlich greifbar“ (DUW 2015:1175) sind und durch das Zutun des Menschen existieren oder sich entwickeln (z. B. vielfarbige Seidenpasins, Stühle, die kleinen Luk Deng = ,Währung in Siam').

10 Die Bezeichnung „Mensch/Eigenschaft/Situation/kulturelle Herkunft“ ist nachfolgend gekürzt zu Mensch/E/S/kH.

11 Die Gliederung von Emotionen übernehme ich von Schwarz-Friesel, die einer offenen Liste von sechs Basisemotionen verschiedenste Sekundäremotionen zuordnet. Zu den Basisemotionen unangenehmer Art zählen Furcht, Trauer, Zorn und Ekel, zu den Basisemotionen angenehmer Art Freude und Liebe (vgl. 2007:67ff., dort eine Auflistung von Sekundäremotionen). 
Unter dem Denotatsbereich NATUR verstehe ich ,alles, was an organischen u. anorganischen Erscheinungen ohne Zutun des Menschen existiert od. sich entwickelt“, es ist „die Gesamtheit der Pflanzen, Tiere, Gewässer u. Gesteine als Teil der Erdoberfläche od. eines bestimmten Gebietes“ (DUW 2015:1252). Das Beispielmaterial zu organischer Natur lässt eine Aufgliederung in Flora und Fauna zu:

(1) Natur/Flora: Hier sind alle Bezeichnungen für die Pflanzenwelt eines bestimmten Gebietes zusammengestellt (z. B. Hibiskusknospen, Zuckerrohrpalmen).

(2) Natur/Fauna: Erfasst sind Bezeichnungen für die Tierwelt eines bestimmten Gebietes (z. B. Falter, Giftschlange).

(3) Natur/anorganisch: Hier sind Bezeichnungen für Denotate erfasst, die zum unbelebten/ anorganischen Teil der Natur gehören, wie die Elemente Luft, Feuer, Wasser, Erde, die Gestirne mit ihrem Licht und ihren Schatten, auch das Klima, wie Wind und Wetter (z. B. die vereinzelten Hügel, ein greller Blitz, glühende Tropenluft).

Aus der Zuordnung aller Bildempfänger und aller Bildspender zu Denotatsbereichen lassen sich einige Erkenntnisse ableiten, die Karlins Umgang mit Vergleichen konkretisieren:

Bei den Bildempfängern sind die drei oben genannten Denotatsbereiche quantitativ unterschiedlich vertreten. In der Gesamtzahl von 133 untersuchten nominalen Vergleichen findet sich der Bildempfänger MENSCH in 82 Vergleichen (=61,65 \%), der Bildempfänger KULTUR in 37 Vergleichen $(=27,81 \%)$ und der Bildempfänger NATUR in 14 Vergleichen $(=10,52 \%)$. Dieses quantitative Verhältnis korreliert mit der Intention Karlins, vor allem die im Roman agierenden Figuren mittels Vergleiche ,anschaulicher“, erfahrbarer zu machen.

In der Gruppe der Bildspender treten die gleichen Kategorien wie im Bildempfängerbereich auf, erweitert um die Kategorien: Übernatürliches ${ }^{12}$ (z. B. wie Lots Weib, wie eine richtende Gottheit, wie eine Segnung), Naturvorgang (Lebewesen betreffende Vorgänge, $\mathrm{z}$. B. wie im Traum, wie im Fieberfrost) und Natur/Zeit (naturbezogene Zeitbegriffe, z. B. wie der hinsterbende Tag).

Für die Konstruktion eines Vergleichs, bestehend aus Bildempfänger und Bildspender, nutzt Karlin bevorzugt semantische Sprünge in heterogenen Vergleichen, d. h., Bildempfänger und Bildspender stammen aus verschiedenen Denotatsbereichen bzw. deren Subkategorien. Mit heterogenen Vergleichen kann Karlin stilistisch relevant die Bildlichkeit des Dargestellten verstärken sowie Rezipienten eindrucksvoll überraschen, wie z. B. Mensch/Gattung $<{ }^{13}$ Natur/anorganisch: Menschen - wie Steine, die im Flußbett gegeneinandergerieben werden (WT 181); Mensch/Beruf/ Stand < Natur/Fauna: der Sultan - wie eine geborstene Schweinsblase (WT 47); Soziale Kultur < Natur/anorganisch: des allgemeinen Lärms des Ostens, der wie der Wellenschlag an rauher Küste kein Abnehmen kennt (WT 49; aE über Siam).

Die wenigen homogenen Vergleiche, d. h., Bildempfänger und Bildspender stammen aus gleichen Denotatsbereichen bzw. gleichen Subkategorien, erhöhen die Bildlichkeit nicht so stark, stilistisch sind sie weniger relevant. Sie überraschen nicht, eher stellen sie die beiden Komponenten mit einer bereichsähnlichen Beschaffenheit semantisch gegenüber, wie z. B. Mensch/Beruf/Stand < Mensch/Beruf/Stand: (d'Albuquerque versteckt sich), bis der Koch, der eben ein Huhn geschlachtet hatte und verfänglich wie ein heidnischer Opferpriester aussah, mit dem blutigen Messer in der Hand verschwunden sein würde (WT 114; aE über d'Albuquerques Angst vor dem Koch). Weitere Vergleiche seien exemplarisch genannt: Mensch/Menschlicher Körper $<$ Mensch/Menschlicher Körper: Zehen - wie Finger (WT 10); Materielle Kultur < Materielle Kultur: komische Wasserhäuser - wie aufgeklappte Holzschachteln (WT 18).

Individualstilistisch markiert sind die (wenigen) Dopplungen des Bildspenders: ${ }^{14}$ Wie Schnee-

12 Mit dem Denotatsbereich Übernatürliches erfasse ich diejenigen Sachverhalte, die für Menschen ,über die Gesetze der Natur hinausgehend u. mit dem Verstand nicht zu erklären“ sind (DUW 2015:1815). Im Wesentlichen handelt es sich im Roman um übernatürliche Wesen aus dem Volksglauben.

13 < zeigt die Richtung der Vergleichskonstruktion an: vom Bildspender zum Bildempfänger.

14 Hier ist erwartungsgemäß der Bildspender analog zum Bildempfänger gedoppelt, das Verb zusammenpassen präsuppo- 
flocken wirbelten weiße Falter über das satte Tropengrün. Auf und nieder, hin und her, wie Elfenfederbälle flogen sie im Lichtmeer des Tropentages (WT 161; aE über die weißen Falter). Die für Siam typischen weißen Falter erhalten durch die Vergleiche mit Bildspendern verschiedener Denotatsbereiche, der anorganischen Natur (Schneeflocken) und des Übernatürlichen (Elfenfederbälle), eine besondere stilistische Dominanz.

\subsection{Exemplarische Analyse zum Denotatsbereich MENSCH - Bildempfänger und Bildspender}

Nachfolgend stelle ich exemplarisch am Denotatsbereich MENSCH Grundsätzliches vor, um Karlins Schreibduktus vertiefter zu verdeutlichen. Der Denotatsbereich MENSCH dominiert als Bildempfänger mit 82 Belegen (von 133 nominalen Vergleichen), was aufgrund der Textbotschaft nicht verwundert, stehen doch die Entscheidungen der literarischen Figuren bezüglich der Partnersuche im Zentrum der Romanhandlung. Die acht Subgruppen der Bildempfänger tangiert Karlin in unterschiedlicher Quantität:

(1) Mensch/Eigenname/Pronomen

(2) Mensch/Menschlicher Körper

(3) $\mathrm{Mensch} / \mathrm{E} / \mathrm{S} / \mathrm{kH}$

(4) Menschliche Emotion

(5) Mensch/Gattung

(6) Mensch/Beruf/Stand

(7) $\mathrm{Mensch} /$ Geschlecht

(8) Menschliche Empfindung in 41 Vergleichen

in 22 Vergleichen

in 6 Vergleichen

in 3 Vergleichen

in 3 Vergleichen

in 3 Vergleichen

in 2 Vergleichen

in 2 Vergleichen

Tab. 2: Bildempfänger-Subgruppen im Denotatsbereich MENSCH

Die Verteilung der Bildempfänger auf die einzelnen Subgruppen offenbart, welche Aspekte des Menschen bzw. der literarischen Figuren die Autorin Karlin als ,vergleichsrelevant“ erachtet. Es dominieren die Eigennamen der Figuren (inklusive der Pronomen) stellvertretend für die literarischen Figuren, deren körperliche Besonderheiten sowie Personenbezeichnungen, die durch eine Eigenschaft, eine bestimmte Situation oder durch die kulturelle Herkunft motiviert sind - eine Reflexion der Romanintention.

Ebenso unterschiedlich wählt Karlin für den gesamten Denotatsbereich MENSCH die Bildspender-Subgruppen aus:

(1) Natur/Fauna

(2) $\mathrm{Mensch} / \mathrm{E} / \mathrm{S} / \mathrm{kH}$

(3) Natur/Flora

(4) Natur/anorganisch

(5) Materielle Kultur

(6) Übernatürliches

(7) Mensch/geschlechtsneutral

(8) Naturvorgang

(9) Mensch/Geschlecht

(10)Mensch/Beruf/Stand

(11)Mensch/Menschlicher Körper in 16 Vergleichen

in 13 Vergleichen

in 11 Vergleichen

in 11 Vergleichen

in 10 Vergleichen

in 7 Vergleichen

in 7 Vergleichen

in 3 Vergleichen

in 2 Vergleichen

in 1 Vergleich

in 1 Vergleich

Tab. 3: Bildspender-Subgruppen im Denotatsbereich MENSCH

Die Autorin entscheidet sich bevorzugt für konventionelle Bildspenderbereiche, wie Natur/ Fauna, Natur/Flora und Natur/anorganisch. Es handelt sich hierbei um jahrtausendealte tradierte

niert ,mehr als 1': sie passen zusammen wie Laus und Kopf (WT 84) (d'Albuquerque über ihre Nichte Sissi und Herbert Lange). 
Vorstellungen von Tieren und Pflanzen, die mit menschlichem Verhalten in Zusammenhang gebracht werden. Dass die Subgruppe Mensch/E/S/kH relativ viele Bildspender stellt (z. B. wie ein Eingeborener, wie ein Asiat, wie ein vom Schicksal windelweich geschlagenes Menschenwrack), ist Textbedeutung und Textbotschaft geschuldet.

Des Weiteren ist für Textbedeutung und -botschaft des Romans relevant, wie häufig eine bestimmte literarische Figur als Bildempfänger ausgewählt wird: Klementine 7-mal, Herbert Lange 6-mal, Fräulein d'Albuquerque 6-mal, Rüdiger (Vater von Anna) 5-mal, Emma 4-mal, Helene 2-mal, Moses Ehrental 2-mal. Alle anderen literarischen Figuren werden jeweils mit einem (1) Vergleich stilistisch porträtiert. Dass Karlin zu relativ vielen literarischen Figuren Vergleiche einsetzt, spricht nicht nur für die Geläufigkeit des Stilelements bei der Autorin, sondern auch für die intendierte figurenbezogene Charakterisierung. Gleichzeitig stehen die fokussierten fünf literarischen Figuren prototypisch in Relation zu Karlins „mahnender“ Textbotschaft, dass „gemischtrassige“ Beziehungen zwischen Mann und Frau ,im Unglück“ enden: Das Schicksal der „Mischlingsfrau“ Klementine endet nach der Ablehnung durch den Deutschen Herbert Lange tragisch mit dem Tod. - Herbert Lange formuliert Karlins Romanintention explizit: „gemischtrassige“ Beziehungen sind ein Verbrechen an der Rasse (WT 172). - Die „Mischlingsfrau“ Fräulein d'Albuquerque benennt belastende familiäre Ereignisse bezüglich von „Mischheiraten“, so dass sie selbst ehe- und kinderlos bleibt. - Der Deutsche Rüdiger steht nach der Heirat mit einer Siamesin für den im Roman beschriebenen sozialen und moralischen Niedergang des „weißen Mannes“ in den Tropen. - Emma schließlich verkörpert die junge, selbstbewusste Deutsche, die aus pragmatischen Gründen die Geliebte eines ,weißen Millionärs" wird, allerdings nur für eine von ihm festgelegte kurze Zeitspanne. ${ }^{15}$ Die Hauptfigur Primula, welche die Auffassung Karlins zum Glücken „reinrassiger“ Beziehungen mit einer Eheschließung zwei sich Liebender höchst positiv realisiert, ist mit nur einer (1) Vergleichskonstruktion versehen. Allerdings dürfen die die literarischen Figuren charakterisierenden Vergleichskonstruktionen nicht überbewertet werden, Karlin setzt zur Figurencharakterisierung verschiedenste Stilelemente ein (vgl. Pohl 2019a zur Figur Emma).

Eine Analyse der Vergleiche zu den exponierten literarischen Figuren enthüllt, dass Karlin Bildspender nutzt, die regulär und konventionell zum Bildempfänger MENSCH auftreten: So werden aus dem Denotatsbereich Natur/Fauna Tiere gewählt, die dem europäischen Leser bekannt sind (wie + Kamel, Katze, Affe, Fisch, Polyp), z. B. sie trat den Boden wie eine Katze, die sich nicht zum Sprung entschließen kann (WT 9; aE über d'Albuquerque). Aus dem Denotatsbereich Natur/Flora sind Pflanzen aus dem Handlungsort des Romans die erste Wahl (wie + Alimanderblüte, Bambus, Lotos), z. B. zwei Mädchen - wie eine Lotosknospe beim ersten Morgenstrahl (WT 25; literarische Figur über Prinzessin Bhinarati und Klementine). Bei Bildspendern aus dem Denotatsbereich Natur/ anorganisch setzt Karlin eine reiche Auswahl ein (wie + Berg, Steine, Sterne, Wolken, Ströme, Lava), z. B. Menschen - wie Steine, die im Flußbett gegeneinandergerieben werden (WT 181; Fräulein d'Albuquerque über das Schicksal).

Bildspender, die für einen Bildempfänger aus dem Denotatsbereich MENSCH weniger konventionalisiert sind, wählt Karlin aus dem Denotatsbereich Materielle Kultur (wie + Würfel, Sonntagshut), z. B. sie fühlte sich wie ein Würfel im Loch (WT 31; aE über Klementine) und aus dem Denotatsbereich Mensch/E/S/kH nach einem für die Autorin typischen Muster: wie jemand, der ...+ Situationsbeschreibung, z. B. Klementine Andersen ging die Suriwongsestraße entlang wie jemand, der die Welt an der Leine hat (WT 78; aE über Klementine) oder: All das (tat Herbert Lange - I.P.) in Bitterkeit, wie jemand, der in Angst und Eile eine verborgene Tür in dunkler Zelle sucht (WT 172; aE über Langes Gedanken zur „Mischehe“) oder: so daß er (Herbert Lange - I.P.) nur ihren Namen zu rufen vermochte wie jemand, von Schmerz überwältigt, nur, Gott! Gott! ‘ zu stöhnen vermag (WT 102; aE über die Liebeserklärung Langes an Emma).

15 Emma ist eine zentrale Figur im Figurenensemble, was sich inhaltlich und sprachlich nachweisen lässt (vgl. Pohl 2019a, b, c; 2020). 


\subsection{Exemplarische Analyse zur Figurencharakterisierung durch Vergleiche}

Da ein Großteil der Vergleiche dem aE zuzuordnen ist, kann die Autorin Karlin über ihn, ohne sie mit dem aE gleichzusetzen, eine Beurteilung der literarischen Figuren bildhaft-charakterisierend einbringen. Folgende Seme/Semkomplexe sind präsupponiert (mit je einem exemplarischen Beleg):

- bei der „Mischlingsfrau“ Klementine: ,noch nicht zu einer Persönlichkeit entwickelt“ (wie ein Kind, WT 36), ,nicht konform mit der Umgebung/Umwelt“ (wie ein Würfel im Loch, WT 31), ,realitätsfern“ (wie eine von Blaubarts Frauen, WT 32; wie ein Buddha, WT 35), andererseits ,selbstbewusst' (wie jemand, der die Welt an der Leine hat, WT 78);

- beim Deutschen Herbert Lange: ,hilflos/ausgeliefert' (wie ein verwundetes Tier, WT 116), ,realitätsfern' (wie im Traum, WT 142), ,in auswegloser Lage' (wie jemand, der in Angst und Eile eine verborgene Tür in dunkler Zelle sucht, WT 172); , verzweifelt' (wie jemand, von Schmerz überwältigt, nur, Gott! Gott! ' zu stöhnen vermag, WT 102);

- bei der „Mischlingsfrau“ Fräulein d'Albuquerque: ,von der Realität in Mitleidenschaft gezogen“ (wie eine vom Tropenschauer breitgeschlagene, braungelbe Alimanderblüte, WT 179), ,realitätsfern' (wie Lots Weib, WT 93), ,zaudernd' (wie eine Katze, die sich nicht zum Sprung entschließen kann, WT 9), ,nicht zu einer reifen Persönlichkeit entwickelt' (wie ein Kind, das einen Ball in das Wasser rollen und fortschwimmen sieht, WT 39);

- beim Deutschen Rüdiger: , angepasst' (wie ein Eingeborener, WT 47; wie ein Asiat, WT 149), , unzivilisiertes Benehmen“ (wie ein Tier, bevor es biß, WT 69), ,äußerliche Eigenschaften durch besondere Umstände verloren gegangen, im Inneren wertvolle Eigenschaften erhalten geblieben' (wie ein Berg, den Regengüsse abgeschürft haben, bei dem man jedoch im schroffen Gestein oft unerwartet auf reiche Edelerzadern stößt, WT 62);

- bei der Deutschen Emma: ,pragmatisch“ (wie alle Frauen [...] Wahrheit ist Ihnen lästig, WT 132).

Die charakterisierenden Vergleiche zielen ab auf das Verhalten der Figuren in bestimmten Situationen bzw. gegenüber anderen Figuren, auf Emotionales, auf Charakterliches, auf geistige Fähigkeiten. Karlin hat die Vergleiche stimmig zur Figurenrolle im Roman ausgewählt - so möchte sie die Hauptfiguren offensichtlich verstanden wissen.

Figurencharakterisierungen bezweckt Karlin auch, indem sie Körperliches als Bildempfänger hervorhebt. Welche körperlichen Eigenheiten und welche literarischen Figuren fokussiert sind, lässt über die Textbedeutung hinaus indirekte Schlüsse hinsichtlich der Textbotschaft zu. Karlin zieht zu ca. fünfzehn verschiedenen Äußerlichkeiten Vergleiche heran. Insbesondere wählt sie den dominierenden Sinneseindruck, der vom Kopf eines Menschen ausgeht, wie Stimme, Mund, Ohren, Augen, Nase, Haare, Mund, Zähne und Gesicht. Dazu sind unterhalb des Kopfes die polaren Bezeichnungen Finger, Beine und Zehen herausgestellt, weiterhin Hand, Herz, Nabelschnur, auffallend häufig die Brüste der siamesischen Frauen. Bedeutsamkeit als Bildempfänger erlangt vor allem das Äußere von Klementines siamesischer Großmutter sowie das der Halbsiamesin Fräulein d'Albuquerque. Bei Klementines Großmutter sind die Brüste akzentuiert, z. B. Wie eingetrocknete Kapokhülsen flogen die Brüste auf und ab (WT 109; aE); das Gesicht, z. B. ein Gesicht wie ein Lederapfel zur Weihnachtszeit (WT 32; aE); der Mund und die Zähne, z. B. ein Mund wie ein Ofenloch, das eben erst frisch mit roten Backsteinen ausgemauert worden war und aus dem, wie verstreute Kohlenstücke, schwarze Zähne hervorragten (WT 32; aE), sowie die Beine, z. B. wie zeitgebeizte Holzkeulen (WT 108; aE). Bei Fräulein d'Albuquerque wählt Karlin als Bildempfänger die Zehen, z. B. die Zehen krampften sich wie Finger in Pein (WT 10; aE); die Finger, z. B. die braunen Finger legten sich wie Spinnenbeine um die Ticalnote (WT 11; aE); die Ohren, z. B. bis ich glaubte, Ohren wie die eines Elefanten zu haben (WT 87; d'Albuquerque über sich bei den Anordnungen zu einer 
Hochzeitsfeier) sowie die Stimme, z. B. Fräulein d'Albuquerque [...] erwiderte mit ihrer lebensmüden Stimme, die wie ein ungeöltes Wagenrad ächzte (WT 87; aE über d'Albuquerque).

\section{Stilistische Funktionen der Vergleichskonstruktionen im Karlinschen Roman}

Die von der Autorin intendierten Absichten, die in den verschiedenen Stilwerten der Vergleichskonstruktionen sedimentiert sind, firmieren als deren stilistische Funktionen. Diese sind zwar relational mit Bezug auf das Werk selbst, die Autorin, die ein Kunstwerk „hergestellt“ hat, und die potenziellen Rezipienten, die eine „Werkhaltung“ einnehmen, beschreibbar, eine absolute Trennschärfe existiert nicht, denn die genannten Komponenten stehen in einem ästhetischen Kommunikationszusammenhang (vgl. Hoffmann 2022, i. Dr.).

\subsection{Zu werk-orientierten stilistischen Funktionen}

(1) Die Textbotschaft vermittelt Karlin im Wesentlichen über den Darstellungsinhalt, die Textbedeutung des Romans: Die Beziehungen zwischen Menschen (bei der Partnersuche) in einer interkulturellen Situation, wie sie sich in Siam in den 1930er Jahren widerspiegeln, sind fokussiert. Damit einher geht die Ordnung von Lexik in den Vergleichskonstruktionen, die zu den Denotatsbereichen MENSCH, KULTUR, NATUR mit weiteren Subgruppen gehört. Die Zuordnung der Vergleiche zu den romanrelevanten Denotatsbereichen gibt Aufschluss über die von Karlin gesetzten Schwerpunkte hinsichtlich ihrer Intention (vgl. Punkt 3.).

(2) Dem europäischen Leser werden mittels Vergleiche die das Werk konstituierenden, wesentlichen Eigenheiten der literarischen Figuren sinnfällig nähergebracht, wobei diese vorgestellt und in Raum und Zeit verortet werden, z. B. Wie man sein Bestes verteidigt, wie man sich aufbewahrt wie einen Sonntagshut (WT 29; Emma über sich selbst). Emma kommt vermutlich aus einem bürgerlichen Haushalt Deutschlands der 1930er Jahre (vgl. Romaninhalt), in welchem man zwischen Alltags- und Sonntagskleidung unterschied. Rezipienten in der Erscheinungszeit des Romans besaßen vermutlich gleiche oder ähnliche Erfahrungen, neuzeitliche Rezipienten müssen soziokulturelles Hintergrundwissen zum Verstehen heranziehen. Zudem handelt es sich hier um einen Bildspender aus dem Erfahrungsbereich der Autorin.

Bei der Figurencharakterisierung konzentriert sich Karlin auf drei wesentliche Aspekte: Sie porträtiert die Figuren vergleichend (a) in äußerlichen Merkmalen: Ja, massig, dunkelbraun wie zeitgebeizte Holzkeulen waren die Beine ... (WT 108; aE über Klementines Großmutter); (b) in ihren jeweiligen Gefühlslagen: Er schien ihr mutlos, beschämt; wie ein Polyp, den die eigenen Fangarme erdrückten (WT 32; aE über Klementines Vater) sowie (c) in ihren sozialen Beziehungen zu anderen Figuren: Wie ein unbeschriebenes Blatt deuchte er sie, und sie fühlte sein Knien vor ihrer unerfaßten Weiblichkeit (WT 102; Emma über Herbert Lange, der sie anbetet). Die pointierte Fokussierung auf siamesische Handlungsfiguren und Situationen in Siam entspricht der Romanintention (vgl. Punkt 3.).

(3) Über die Vergleiche-Charakterisierung siamesischer Menschen, ihrer Kultur, ihrer Natur und ihres Glaubens an Übernatürliches in den 1930er Jahren zeichnet Karlin vor allem Lokal- und Zeitkolorit, wodurch die Romanhandlung in eine historische Kulisse gestellt wird. So wird Lokalkolorit durch Bildspender-Benennungen von exotischen Pflanzen und Tieren skizziert: Helene fühlte sich wie ein Bambus im Sturm (WT 57; aE über Helene, als sie von den Heiratsabsichten des deutschen Witwers Sturpopp erfährt). Bambus ist vor allem in Südostasien beheimatet. Weitere lokalkolorierende Bildspender sind z. B.: Rüdiger - wie ein Affe vor, nem Tiger (WT 47); schlanke Gestalt wie ein Bambusrohr im Wind (WT 82); Finger beider Hände - wie Fächerpalmenwedel (WT 87); Frauenherz - wie eine Alimanderblüte im Morgensturm (WT 165); eingeschrumpfte Brüste - wie 
eingetrocknete Datteln (WT 32); sein Blick (der des Kochs - I.P.) - wie der Blick eines Tempeltorgottes (WT 138); Silberschüsselchen - wie sich öffnende Lotos (WT 50).

Exotische Bildempfänger mit lokalkolorierender Semantik werden mit alltäglichen, den europäischen Lesern bekannten Bildspendern veranschaulicht: die weiten Zweige einer Bougainvillia wie ein abgeworfener Bischofsmantel (WT 60); die roten Hibiskuskelche - wie Festmahlsbecher (WT 174); Hibiskusknospen - wie weiche, rote Finger, die eine Fee prüfend in eine unbekannte Welt streckte (WT 91); Blütenregen der Flammenmimosen - wie ein Meer brennender Herzen (WT 101); erste Zuckerrohrpalmen - wie stolze Wächter mit grünen Federbuschhelmen (WT 173); die vereinzelten Hügel - wie angebissene und vergessene Tortenstücke auf der weiten Ebene bewässerter Reisfelder (WT 144); glühende Tropenluft - wie eine brütende Henne (WT 31).

Wenige Bildspender kolorieren mittels gesellschaftlicher Gepflogenheiten die Zeit des beginnenden 20. Jahrhunderts (Trennung von Werktags- und Sonntagskleidung, Modalitäten des Ofenbaus, Wander-Scherenschleifer): Wie man sein Bestes verteidigt, wie man sich aufbewahrt wie einen Sonntagshut (WT 29); ein Mund wie ein Ofenloch, das eben erst frisch mit roten Backsteinen ausgemauert worden war und aus dem, wie verstreute Kohlenstücke, schwarze Zähne hervorragten (WT 32); die Sätze - wie ein gutgewässerter Schleifstein (WT 85).

\subsection{Zu autorin-orientierten stilistischen Funktionen}

(1) Die Autorin kann über die Vergleiche in der Stimme des aE ihre Meinung über literarische Figuren, Ereignisse usw. bildhaft einbringen, wobei eine differenzierte Wertung erkennbar ist: Über Siam im Allg. kommt eine negative Wertung zum Ausdruck, die semantisch in den Bildspendern mit pejorativen Semen eingefangen ist: So vergleicht der aE den allgemeinen Lärm des Ostens mit Wellenschlag an rauher Küste, der kein Abnehmen kennt (WT 94); die östliche Umgebung mit erstickender frischer Erde auf zu junge Keime (WT 97); den Osten mit seinen tausend bunten Lichtern mit einem Bann, dem niemand entgeht (WT 157). Andererseits hat der aE Hochachtung vor den kulturellen Eigenheiten des Landes (z. B. Münzen, Bekleidung, materielle, von Menschen hergestellte Güter), die sich in positiv-emotiven Semen der Bildspender zeigt: die kleinen Luk Deng aus dem Straßenstaub der Suriwongse vergleicht er mit leuchtenden zerstreuten Korallen (WT 113); weiterhin sind gegenübergestellt vielfarbige Seidenpasins - wie windgetragene Blütenblättchen (WT 59); Silberschüsselchen - wie sich öffnende Lotos, sich dem Winde ergebende Blättchen oder durchgeschnittene Äpfel (WT 50). Aus zahlreichen Vergleichen mit positiv-emotiven Semen spricht die Bewunderung der Natur in Siam. Karlin entfaltet die siamesische Pflanzenwelt vor dem inneren Auge des Rezipienten, indem sie die Schönheit von Bougainvillia, Hibiskusknospen, Hibiskuskelchen, Flammenmimosen, Zuckerrohrpalmen über positiv wertende Lexeme in den Vergleichen verbalisiert, z. B. erste Zuckerrohrpalmen erscheinen wie stolze Wächter mit grünen Federbuschhelmen (WT 173). Insbesondere beeindrucken die weißen Falter in Siam (vgl. Aufsatzanfang), welche die Autorin mit mehreren Vergleichen bewundernd heraushebt: nur die Falter [...] wirbelten nach wie vor als große weiße Flocken, wie Maienschnee vorüber, und mit ihrem allmählichen Verflattern kam unerbittlich der Abend (WT 165; aE über Klementines Sterbestunde). Eine durchaus positive Wertung wird auch der anorganischen Natur entgegengebracht: So starrte sie auch jetzt auf die vorbeisausende Landschaft hinaus - auf die kerzengeraden Zuckerpalmen mit ihren Rotanleitern, die vereinzelten Hügel, die wie angebissene und vergessene Tortenstücke auf der weiten Ebene bewässerter Reisfelder [...] verstreut waren (WT 144; aE über Emmas Reise mit Moses Ehrental).

(2) Die relativ hohe Zahl an „veranschaulichenden“ Vergleichen i. w. S. ist charakteristisch für Karlins individuellen Stil. Die quantitative Salienz (vgl. Punkt 2.) wird durch einige Besonderheiten unterstützt. So kehren in mehreren Vergleichskonstruktionen Bildspender wieder: wie im Traum (2x) und wie ein Kind (7x), denen man aufgrund des Wiederholungseffekts Topikcharakter zusprechen kann, z. B. Klementine folgte wie im Traum, stolperte dahin, ohne Hindernisse zu beachten (WT 
148; aE über Klementine bei der Suche nach Anna, nachdem Herbert Lange sie abgelehnt hat). Der Bildspender wie ein Kind wird nachweislich nicht bei den literarischen Figuren Emma, Rüdiger und Moses Ehrental genutzt, möglicherweise hat Karlin diese literarischen Figuren bewusst als in einer gewissen Weise ,gereift/vom Kindsein entfernt' angelegt.

(3) Auffällig sind in vielen Vergleichskonstruktionen die Lexemanhäufungen sowohl beim Bildempfänger als auch beim Bildspender (,erweiterte Vergleiche“), mitunter im gleichen Satz. Diese formale Eigenheit ermöglicht der Autorin, detailreiche Bilder zu schaffen und die Wirkung ihrer Gedanken beim Rezipienten zu vertiefen.

Unter anderem zieht dieselbe literarische Figur im gleichen Satz mehrere Vergleiche auf sich: Fräulein d'Albuquerque spreizte die Finger beider Hände wie Fächerpalmenwedel auseinander, zuckte die Achseln, seufzte, erwiderte mit ihrer lebensmüden Stimme, die wie ein ungeöltes Wagenrad ächzte (WT 87; aE über d'Albuquerque). Auch die Bezeichnung eines Körperteils (Finger) kann mehrere Bildspender und mehrere Informationen akkumulativ auf sich vereinigen: spottete offen über die Finger, die wie Fischgräten vorstanden und sich nicht wie Halme zurücklegen ließen (WT 155; aE über die Verspottung Bhinaratis seitens einer Zweitfrau ihres Vaters). Stilistische Relevanz wird durch die Akkumulation der Informationen erreicht. Auch derselbe Sachverhalt (Tropenluft) kann im gleichen Satz mehrfach vergleichend charakterisiert sein: Die glühende Tropenluft, die wie etwas greifbar Schweres, Feindliches auf Bangkok herabgedrückt hatte, hob sich langsam wie eine brütende Henne, um den kühlenden Abendwind hindurchwehen zu lassen (WT 31; aE über Bangkok).

Häufiger sind es verschiedene Bildempfänger (Blitze, Herz) und deren Bildspender, die umfangreiche Satzkonstruktionen entstehen lassen: In der Ferne zuckten Blitze wie Schwerter in erfahrenen Händen [...] doch nur Frau Langfar fühlte es, denn ihr Herz war wie eine Wunschtrommel, die ununterbrochen vertrauensvoll läutete; sie sang ,Primula--Pri-mu-la!“(WT 181; aE über die Missionarsfrau Langfar).

Schließlich bringen Vergleiche verstärkende Fortschreibungen in benachbarten Sätzen (zur Charakterisierung des Deutschen Rüdiger): Klementine Andersen merkte, daß er (Rüdiger - I.P.) anders war als sonst, wie von einer stillen Wut getragen oder einem verborgenen Leid bis zur Tollheit des Schmerzes gequält; einer Wut gegen irgend etwas - irgend jemand-unter der, wie Lava unter auffliegendem Rauch und Gestein, ein glühender Kummer brannte ... Wie mit einem Messer eingehackt, schienen heute die Züge des rotbraungebrannten, tropengegerbten Gesichts (WT 69; Klementine über Rüdiger). Bei derartigen wortreichen Akkumulationen muss man fragen, ob es nicht „ein Zuviel“" des Stilelements Vergleich ist und wo eine Grenze zum Kitsch überschritten ist. Eine Antwort kann nur in Relation zu den Lesebedürfnissen des Leserpublikums abgewogen werden. Dass Karlins Schreibstil offensichtlich einen Nerv des Zeitgeschmacks traf, zeigt die dreimalige Auflage des Romans im gleichen Erscheinungsjahr.

(4) Modifikatorische Besonderheiten im Karlinschen Sprachgebrauch lassen sich an denjenigen Vergleichen ausmachen, deren Komponenten Phraseologismen nahestehen.

Beispielsweise ergänzt Karlin einen Vergleich zu einem idiomatisierten Verb, so dass dessen Bedeutung „entidiomatisiert“ visualisiert wahrgenommen werden kann: „Sie werden wie eine Rakete hochgehen, doch dazu ist keine Veranlassung vorhanden, denn was ich da vorschlage, braucht Sie weder zu verletzen noch aufzuregen! " (WT 132; Moses Ehrental macht Emma den Vorschlag, seine zeitlich begrenzte Geliebte zu werden). Das Verb hochgehen (ugs.) trägt die idiomatisierte Bedeutung ,in Wut, in Zorn geraten' (DUW 2015:876), möglicherweise mit einer körperlichen Reaktion, wie vom Platz aufstehen, verbunden. Indem Karlin den wie-Vergleich wie eine Rakete anfügt, wird gleichzeitig die wörtliche Bedeutung von hochgehen inferiert: , sich nach oben, in die Höhe bewegen' (DUW 2015:876), so dass die emotive Bedeutung von hochgehen visualisiert wird.

Auffällig substituiert Karlin Lexik in vorhandenen Mustern komparativer Phraseologismen. Das Muster sie passen zusammen wie ein Paar/wie zwei alte Latschen/wie Topf und Deckel bedeutet 
auf Menschen bezogen: (ugs.) ,sehr gut zusammenpassen“ (DuR 2008:915). Karlin substituiert den Bildspender: sie passen zusammen wie Laus und Kopf (WT 84). Die phraseologische Grundbedeutung bleibt bestehen, jedoch präsupponiert die substituierte Komponente den ,soziokulturellen Hintergrund“ der Romanhandlung.

Karlin gebraucht außerdem Phraseologismen in wörtlicher Bedeutung, jedoch ist die Inferenz zu einem vorhandenen Sprichwort mit phraseologischer Bedeutung präsupponiert, z. B. daß der Name ,Lenchen' sie deckte; vollständig, wie ein Deckel den Topf (WT 6; Emma über Helenes Vornamen). Zugrunde könnte das Sprichwort jeder Topf findet seinen Deckel liegen, mit der Bedeutung,für jeden findet sich ein passender Lebenspartner' (DuR 2008:778), das hier form- und semantikmodifiziert (ein Onym als Bildempfänger) eingesetzt ist.

Schließlich ersetzt Karlin Komponenten eines etablierten komparativen Phraseologismus durch eine Paraphrase. Als Basis dient z. B. der Phraseologismus sich fühlen wie ein Fisch auf dem Trockenen mit der Bedeutung, sich hilflos, von seinem Lebenselement abgeschnitten fühlen“ (DuR 2008:249). Karlin transformiert die Form (Wortgruppe > Satzgliedteilsatz), die phraseologische Bedeutung bleibt erhalten: Primula schnappte dreimal nach Luft, wie ein Fisch, den die Springflut an den Strand geworfen (WT 152, aE über Primula, nachdem sie erfahren hat, dass die Halbsiamesin Anna nach der Heirat eines Malaien nicht Erstfrau, sondern Zweitfrau geworden ist).

(5) Stilistisch relevant sind die Karlinschen Vergleichskonstruktionen auch deshalb, weil die Autorin überwiegend okkasionelle Vergleiche kreiert. Keiner der eingesetzten Vergleiche ist mit gleicher Lexik in einem Wörterbuch aufgeführt. Sie bedient sich zwar konventioneller Bildspendebereiche (vgl. oben Denotatsbereiche, Punkt 3.), jedoch ist die lexematische Ausfüllung im Einzelnen nicht erwartet, unkonventionell, überraschend. Für wenige der von Karlin genutzten Verben sind Listen von konventionellen Vergleichskonstruktionen lemmatisiert, z. B. für das Verb sich fühlen: wie ein Fisch im Wasser, wie die Made im Speck, wie ein Fisch auf dem Trockenen, wie neugeboren, wie durch den Wolf gedreht usw. (vgl. DuR 2008:249). Im Roman äußert sich der aE zur Verfasstheit der Halbsiamesin Klementine: sie fühlte sich wie ein Würfel im Loch (WT 31). Dieses leicht vorstellbare, aber unkonventionelle Bild mit einem Bildspender aus dem Denotatsbereich der Materiellen Kultur metaphorisiert Klementines emotionale Zerrissenheit, dass sie sich in ihrer Umgebung, menschlich unpassend' fühlt, es gibt ,keine Stimmigkeit, keine Symbiose' zwischen ihr und der siamesischen Heimat. Klementines ,unsichtbare“ Gefühlslage wird mit dem okkasionellen Vergleich visualisiert.

Häufig nutzt Karlin z. B. zum Bildempfänger MENSCH konventionelle Bildspender; die Okkasionalität kommt erst im nachfolgenden Nebensatz bzw. in akkumulativen Nebensätzen zum Ausdruck. So werden aus dem Denotatsbereich Natur/Fauna (Kamel, Katze, Affe, Fisch) Tiere gewählt, die dem europäischen Leser bekannt sind und mit denen oft Menschen verglichen werden. Karlin wählt jedoch überraschende, nicht etablierte Vergleichsmerkmale: sie trat den Boden wie eine Katze, die sich nicht zum Sprung entschließen kann (WT 9; aE über d'Albuquerque). Oder aus dem Denotatsbereich der anorganischen Natur (Berg): Äußerlich war er wie ein Berg, den Regengüsse abgeschürft haben, bei dem man jedoch im schroffen Gestein oft unerwartet auf reiche Edelerzadern stößt (WT 47; aE über Rüdiger).

Schließlich findet sich bei Karlin im Prädikativ das Pronomen jemand mit angeschlossenem Nebensatz, das eine Einordnung in eine unkonventionelle Begriffsklasse ausdrückt: Sie war wie jemand, der ganz in einer anderen und in keiner erfreulichen Welt weilte (WT 68; aE über Anna). Diesen Bauplan wie jemand, der ... + Situation verwendet Karlin häufiger, so dass man von einem Topos sprechen könnte, z. B. Klementine-wie jemand, der die Welt an der Leine hat (WT 78); Lange - wie jemand, der in Angst und Eile eine verborgene Tür in dunkler Zelle sucht (WT 172); Lange - wie jemand, von Schmerz überwältigt, nur, Gott! Gott! ‘ zu stöhnen vermag (WT 102). Der usuelle bildspendende Denotatsbereich Mensch/E/S/kH erhält seine Okkasionalität aufgrund der von Karlin ausgewählten individuell-spezifischen Situation. 


\subsection{Zu rezipienten-orientierten stilistischen Funktionen}

(1) Inwieweit ein Rezipient die Vergleiche nachvollziehen kann, ist weitestgehend von der Wahl des Bildspenders abhängig und davon, wie eine relative Nähe-Beziehung zum Rezipienten verbal ausgestaltet wird. Die Anforderungen an Romanrezipienten zum Erschließen der Vergleichskonstruktionen sind m.E. nur mäßig hoch, da die Bildspender zumeist aus usuellen Denotatsbereichen gewählt und nicht ,weit hergeholt“ sind (vgl. Punkt 3.).

Eine starke Rezipientenorientierung erreicht Karlin dadurch, dass Gefühle einer literarischen Figur mit dem Rezipienten geteilt werden. Beim nachfolgenden Beispiel befinden sich der aE und der Rezipient auf gleicher emotional-situativer Ebene, für beide gilt: ,sich in einer bestimmten Lage befinden; sich bestimmten Umständen ausgesetzt sehen' (DUW 2015:1596): Klementine war es, wie es einem manchmal ist, wenn man an einem kalten Wintertag plötzlich die windstille Sonnenfront eines Hauses gefunden ... (WT 36; aE über Klementine nach dem freundlichen Gruß der siamesischen Großmutter). Der Rezipient kann Klementines Zustand wie seinen eigenen empfinden.

Auch wenn okkasionelle Vergleiche gewählt sind, ist das Verstehen häufig mit Hilfe textinterner Relationen problemlos möglich, vgl. sie passen zusammen wie Laus und Kopf (WT 84). Im DuR (2008:915) finden sich zum Verb zusammenpassen die Phraseologismen zusammenpassen wie ein Paar/wie zwei alte Latschen (ugs.); zusammenpassen wie Topf und Deckel (ugs.) mit der Bedeutung ,sehr gut als Paar zusammenpassen'. Für Rezipienten ist der Roman-Vergleich aufgrund des bekannten Musters passen zusammen wie und aufgrund der textlichen Isotopie leicht nachvollziehbar - im Roman sind wiederholt Kopfläuse in der siamesischen Umgebung beschrieben. Hier integriert Karlin einen Sachverhalt aus dem Erfahrungsbereich der literarischen Figur d'Albuquerque, was gleichsam zu deren Authentizität beiträgt.

(2) Vergleichskonstruktionen stellen im Lesefluss Haltepunkte für Rezipienten dar. Sie benötigen Zeit, um dem Vergleich nachzuspüren, die Beziehung zwischen Bildempfänger und Bildspender wahrzunehmen. Gleichzeitig bewirken die detailreichen Vergleichskonstruktionen eine Auflockerung des Textes. Der Wechsel von komplexeren Satzgliedern, wie der zahlreichen Vergleichskonstruktionen, und von kürzeren Satzgliedern unterstützt die formale Auflockerung, z. B. (Satzglieder in eckige Klammern gesetzt): [Die östliche Umgebung] [hatte sich] [in den verrauschenden Wochen] [erstickend] [auf Tup Tim] [gelegt] [wie frische Erde auf zu junge Keime] (WT 97; aE über die siamesische Prinzessin Bhinarati).

Gleichzeitig dient die textliche Gestaltung von Isotopieketten mit Vergleichsstrukturen der semantischen Auflockerung, indem der Text nicht das relativ strenge narrative Isotopienetz ohne „Störung“ bzw. ohne Pause weiterverfolgt. Am o. g. Beispiel ufert eine bestehende Isopiekette zu östliche Umgebung zu einer Subisotopie aus: wie frische Erde auf zu junge Keime, kehrt jedoch im nachfolgenden Textverlauf wieder zum bestehenden Strang „Lebensweise in Siam“ zurück.

(3) Durch die Wahl des Bildspenders wird der Bildempfänger den Sinnen der Rezipienten zugänglicher, sinnfällig gemacht. Die überwiegende Zahl der Vergleichskonstruktionen wählt die Richtung vom Einzelnen, vom Speziellen, vom Unbekannten zu Bildspendern, die dem Rezipienten begreifbar sind, die er mit seinen Sinnen erfassen kann (vgl. Punkt 3.).

(4) Die gewählten ungewöhnlichen Vergleichskonstruktionen bewirken Unterhaltsamkeit, denn Karlin geht spielerisch mit dem Sprachmaterial um, indem sie nicht erwartbare Vergleiche eingängig konstruiert. Für Rezipienten entsteht der Leseanreiz einerseits dadurch, dass er dem Vergleich nachsinnen und ihn auf eigene Lebensumstände beziehen kann. Andererseits sind Rezipienten bei der Lektüre von Erwartungen gesteuert, so erwarten sie prototypische Vergleiche, wie z. B.: sich fühlen wie ein Fisch im Wasser usw. Wird die Erwartung nicht erfüllt, wie bei Helene fühlte sich wie ein Bambus im Sturm (WT 57; aE über Helene, als sie von den Heiratsabsichten des deutschen Witwers Sturpopp erfährt), ist ein Rezipient anregend-unterhaltend herausgefordert. Er muss inferierend herausfinden, dass mit dem Bildspender der schnell wachsenden Graspflanze, die einen leichten, hohlen 
Stängel besitzt und vom Wind leicht hin und her bewegt werden kann (vgl. DUW 2015:253), die schwankenden Gefühle Helenes visualisiert werden.

Unterhaltsam sind viele der Karlinschen Vergleiche auch deshalb, weil sie über die ästhetische Kategorie des Komischen verfügen. Grundlegend lässt sich das Komische als Konflikt widersprüchlicher bzw. ungleichwertiger Prinzipien verstehen. Menschen lachen, weil sie die ungleiche Konfrontation entdeckt haben, sie identifizieren sich mit dem überlegenen Prinzip im Glauben daran, dass dem Unterlegenen kein ernsthafter Schaden entstanden ist. Innerhalb eines inferenztheoretischen Rahmens lassen sich die folgenden Vergleiche erläutern: Und sie - armes Weib - hatte Augen wie ein Albinokaninchen und eine Nase wie eine eben zum Salat angeschnittene Runkelrübe (WT 89; de'Albuquerque über die weinende Helene, deren Hochzeit vom Bräutigam abgesagt wurde). Unsere kognitive Erfahrungswelt als Erwartungsstruktur beinhaltet zum Sachverhalt einer abgesagten Hochzeit, dass die potenzielle Braut stark weint, dass Augen und Nase deshalb gerötet sein werden (= Erfahrungswelt 1). Komisches entsteht dadurch, dass sich durch die Bildspender eine unerwartete Erfahrungswelt 2 (Albinokaninchen, Runkelrübe) in Konflikt zur Erfahrungswelt 1 befindet und beide Erfahrungswelten mit Bezug auf den Text bestehen bleiben (vgl. Pohl 1997:346). Im Verständnis der von Sigmund Freud beschriebenen Theorie der „Aufwanddifferenz“ erscheint uns derjenige komisch, ,,der für seine körperlichen Leistungen zu viel und für seine seelischen Leistungen zu wenig Aufwand im Vergleich mit uns treibt" (Freud 1970:182) - das Albinokaninchen und die Runkelrübe sind innerhalb eines möglichen Vergleichsframes ,eine Prise“ zu viel, der weinenden Helene gegenüber fühlen wir uns als Rezipienten ,lustvoll“ überlegen. Die Widersprüchlichkeit, die zu Komik führt, lässt sich auch am folgenden Beispiel zeigen. Die literarische Figur Emma will Herbert Lange heiraten, wobei sie aus verschiedensten Gründen große Bedenken hat und traurig über ihre Zukunft nachdenkt. Die Freundin Helene benennt ironisch die glückliche Braut und setzt deren unglückliches Gesicht in den Zusammenhang eines Hinrichtungsrituals: „Für eine glückliche Braut machst du ein Gesicht wie ein Verurteilter, dem der oberste Henkersknecht eben Lehm in Nase und Ohren stopft, bevor er den letzten Schwerttanz beginnt" (WT 115; Helene zu Emma, die Lange heiraten will). Das Ironisch-Hyperbolische steht im Widerspruch zu einer normal-menschlichen Situation der Brautwerbung, in welcher der Umworbenen kein ,ernsthafter Schaden“ droht, so dass sich bei Rezipienten ein vergnügliches Überlegenheitsgefühl einstellen kann. Im semantischen Widerspruch zum Hinrichtungsritual steht zudem der Schwerttanz, der ursprünglich einen „Waffentanz von Männern mit gezogenen Schwertern“ (DUW 2001:1585) meint und zu einer Gruppe von Volkstänzen gehört.

\section{Fazit}

Am polyfunktionalen Stilelement der Vergleichskonstruktion erweist sich die sprachliche Kreativität Karlins. Der Autorin kann ein umfangreiches Reservoir vergleichender Ausdrucksformen bescheinigt werden, welches sie literar-ästhetisch romanrelevant einsetzt. Sie nutzt das vorhandene syntaktisch-strukturelle Modell der Vergleichskonstruktion und füllt dieses individuell mit nicht erwarteter, okkasioneller Lexik, auch wenn sich feststellen lässt, dass Karlin keine ungewöhnlichen, etwa überraschenden Relationen zwischen den Denotatsbereichen von Bildempfängern und Bildspendern wählt.

Die stilistische Relevanz der Vergleichskonstruktionen ergibt sich aus ihrer Quantität, insbesondere der ,veranschaulichenden“ Vergleichskonstruktionen, besonders jedoch aus ihrer Qualität. So kann demonstriert werden, dass Karlin bevorzugt heterogene Vergleiche einsetzt, welche der „Sinne-Ansprache“ des Rezipienten entgegenkommen. Die spezifischen stilistischen Funktionen der Vergleiche im Romantext lassen sich umreißen mit Sinnfälligkeit (bei besonderer Bevorzugung des optischen Sinns), Figurencharakterisierung, Unterhaltsamkeit bis hin zu Komik, Auflockerung, Historizität, Rezipientenzugewandtheit; die Vergleiche geben der Autorin Stimme und vermitteln Lokal- und Zeitkolorit des Darstellungsinhaltes. Zur Karlinschen Textbotschaft lassen sich keine Vergleiche finden, was man als individuelles stilistisches Merkmal betrachten kann. 
Individualstilistische Eigenheiten in der literar-ästhetischen Gestaltung des Romans sehe ich darin, dass die Autorin zum einen bevorzugt quantitativ reich ausgestaltete Vergleichskonstruktionen („erweiterte Vergleiche“) sowie Vergleichshäufungen („Vergleiche-Akkumulationen“) einsetzt, die zu semantischem Mehrwert bei den Bildempfängern führen. Zum anderen erweist sich die unterhaltend-spielerische Modifikation vorhandener phraseologischer Komponenten von Vergleichen als wesentliches Element Karlinscher Sprachbewusstheit.

\section{Literaturverzeichnis}

\section{Primärliteratur:}

Karlin, Alma M. (1933): Windlichter des Todes. Roman aus Siam. 3. Aufl. Leipzig.

\section{Sekundärliteratur:}

Burger, Harald (2007): Phraseologie. Eine Einführung am Beispiel des Deutschen. Berlin.

DuR 2008 (2008) = Dudenredaktion (Hrsg.): Duden. Redewendungen. Mannheim, Zürich.

DUW 2015 (2015) = Dudenredaktion (Hrsg.): Deutsches Universalwörterbuch. Berlin.

Fleischer, Wolfgang / Michel, Georg / StARKe, Günter (1993): Stilistik der deutschen Gegenwartssprache. Frankfurt am Main u. a.

Freud, Sigmund (1970): Der Witz und seine Beziehung zum Unbewußten (1905). Frankfurt am Main. Hoffmann, Michael (2022, i. Dr.): Textorientierte Wortschatzarbeit. In: PoHL, Inge / UlRICH, Winfried (Hrsg.): Wortschatzarbeit. Baltmannsweiler.

PoHL, Inge (1997): Textsemantisierung: Inferenzen über Inferenzen. In: PoHL, Inge (Hrsg.): Methodologische Aspekte der Semantikforschung. Frankfurt am Main u. a., S. 337-364.

PoHL, Inge (2019a): Epische Darstellungstechniken in Alma M. Karlins „Windlichter des Todes. Roman aus Siam“. Eine textlinguistisch-stilistische Analyse. In: JESENŠEK, Vida / EHRHARDT, Horst (Hrsg.): Sprache und Stil im Werk von Alma M. Karlin. Maribor u. a., S. 137-174.

PoHL, Inge (2019b): Ungewöhnliche Wortbildung am Beispiel von Okkasionalismen in Alma M. Karlins Roman „Windlichter des Todes. Roman aus Siam“. In: JESENŠEK, Vida / EHRHARDT, Horst (Hrsg.): Sprache und Stil im Werk von Alma M. Karlin. Maribor u. a., S. 259-293.

PoHL, Inge (2019c): Kodierung von Emotionen in ,Windlichter des Todes. Roman aus Siam‘ von Alma M. Karlin. In: Acta Facultatis Philosophicae Universitatis Ostraviensis. Studia Germanistica, Nr. 24, S. 31-46.

PoHL, Inge (2020): Zur sprachlichen Kreativität Alma M. Karlins - nachgewiesen an Phraseolexemen und satzwertigen Phraseologismen in „Windlichter des Todes. Roman aus Siam“ (1933). In: Acta Facultatis Philosophicae Universitatis Ostraviensis. Studia Germanistica, Nr. 27, S. 47-71.

SAndig, Barbara (2006): Textstilistik des Deutschen. Berlin; New York.

Schwarz-Friesel, Monika (2007): Sprache und Emotion. Tübingen; Basel.

SKIRL, Helge / SchwARZ-Friesel, Monika (2007): Metapher. Heidelberg.

WeInRICH, Harald (1993): Textgrammatik der deutschen Sprache. Mannheim u. a. 